\title{
Intraocular pressure measurement at the choroid surface: a feasibility study with implications for implantable microsystems
}

\author{
Raed N Rizq, Woo-Hyek Choi, David Eilers, Martha M Wright, Babak Ziaie
}

\begin{abstract}
Aims-To demonstrate that a sensor, which is inserted through the sclera and placed in intimate contact with the choroid, can reliably detect changes in the intraocular pressure (IOP).

Methods-A manometer was used to control the IOP of three cadaver eyes in steps of $7 \mathrm{~mm} \mathrm{Hg}$. A piezoresistive pressure sensor was used to measure the pressure at the choroid through a $2.5 \mathrm{~mm}$ diameter hole that was surgically removed from the sclera. Data were collected for two configurations; with the sensor: (i) rigidly attached to a miniature positioning stage, and (ii) sutured to the sclera.

Results-Both configurations accurately tracked the manometer pressure from 10 $\mathrm{mm} \mathrm{Hg}$ to $47 \mathrm{~mm} \mathrm{Hg}$. For the fixed sensor cases, the average difference between the pressure measured at the choroid and in the anterior chamber was $0.8 \mathrm{~mm} \mathrm{Hg}$ for the three eyes. For the sutured sensor case, the average difference was $2.1 \mathrm{~mm}$ $\mathrm{Hg}$-although a significant portion of this was attributed to an initial offset. The standard deviations at each pressure level for all of the choroid measurements were under $1.0 \mathrm{~mm} \mathrm{Hg}$.

Conclusions-Small changes in IOP can be accurately measured by a sensor in contact with the surface of the choroid, for both a fixed sensor configuration and for a sensor sutured to the sclera. These results are the first step in the realisation of a surgically implantable microsensor to monitor IOP for patients suffering from low tension and other difficult to manage forms of glaucoma.
\end{abstract}

(Br F Ophthalmol 2001;85:868-871)

Continuous non-invasive measurement of intraocular pressure (IOP) over several days or months would be extremely helpful in accurate diagnosis and management of glaucoma; this is especially true for low tension glaucoma, or in cases of unexplained progression despite reaching target pressures in the physician's office. Manometry (introduction of a catheter inside the eye) and tonometry (indirect measurement by applanation of the cornea) have been the standard techniques for measuring IOP over the past two centuries. ${ }^{1}$ However, they are not suitable for continuous noninvasive IOP measurement (manometry is invasive and traditional applanation tonometry provides only discrete readings). Radiotelemetry is an attractive method for obtaining continuous pressure measurements without hardwire connections. Over the past three decades, there have been numerous efforts to develop miniature pressure or strain transducers for wireless IOP measurement (either implanted inside the eye or incorporated into contact lenses)..$^{2-5}$ Most of these systems have consisted of passive components (usually an inductor capacitor (LC) tank circuit) whose resonant frequency shift caused by pressure variations were monitored by using various radiofrequency (RF) techniques. These efforts have not been successful and suffered from several shortcomings, which so far have prevented their widespread application. These include: (1) short range and poor accuracy due to small signal to noise ratio in passive transmission, (2) poor accuracy and long term stability due to unreliable mechanical contact with the sclera (this is particularly serious in the transducers imbedded in a contact lens), (3) high profile $(>1 \mathrm{~mm}$, therefore, making them unsuitable for surgical implantation into the sclera), and (4) high manufacturing costs due to hand assembly and packaging.

In order to achieve continuous, long term, and reliable monitoring of IOP and overcome the above shortcomings, research is currently under way to develop a low profile $(<1 \mathrm{~mm}$ thick) implantable micromachined piezoresistive pressure transponder, fabricated with associated electronics (interface circuit, RF powering, and reverse telemetry). An active readout technique (that is, with full on-board electronics) has been selected which provides a superior signal to noise ratio, range, and accuracy compared with previous passive techniques. A portable transceiver approximately the size of a deck of cards will be constructed to power the microsystem and receive the measured IOP signal through a spectacle mounted antenna.

One of the key elements of the above work is to identify a surgically feasible location in which to implant the sensor/transponder chip. This location has to satisfy the following requirements: (1) provide a surgically feasible location for easy access and for minimally invasive placement and retrieval, and (2) enable accurate, reliable, and unobtrusive measurement of the IOP. A small hole $(<3 \mathrm{~mm}$ in diameter) trephined into the sclera may provide an ideal location for implantation of the pressure measurement system (Brubaker RF, Mayo Clinic and Foundations, private 


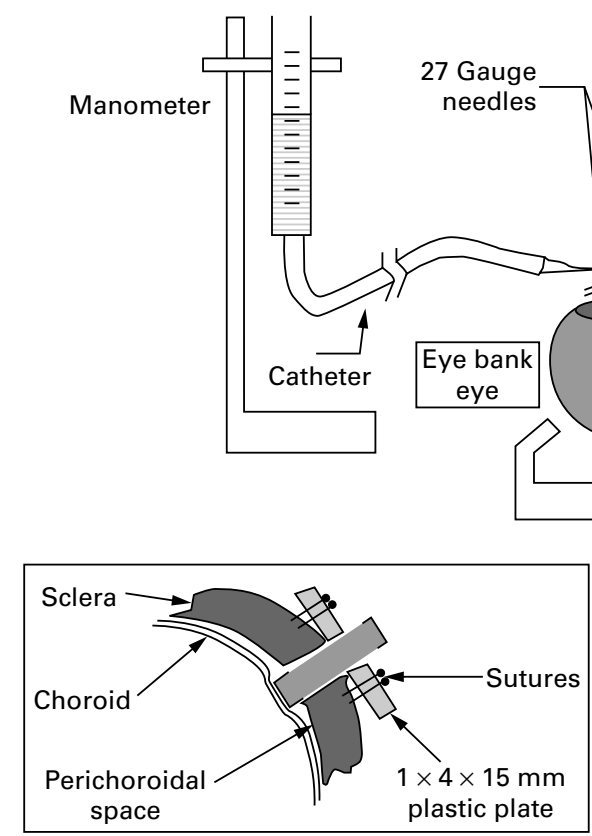

Entran pressure transducer (choroid) $x-y-z$ Miniature positioning stage

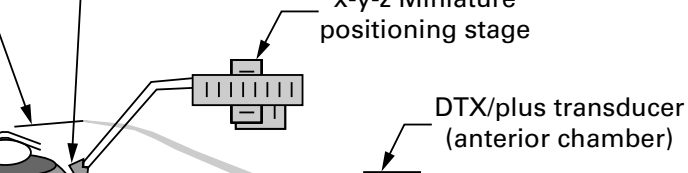

Figure 1 Experimental apparatus used to set the anterior chamber pressure and to monitor the IOP at the choroid surface and the anterior chamber.

communication). This implantation technique is minimally invasive and may improve accuracy by removing the tough fibrous scleral tissue and placing the transducer in intimate contact with the choroid. In the following sections, we will discuss the measurement results from several experiments, which were conducted in order to investigate the accuracy and reliability of transchoroidal IOP measurements.

\section{Materials and methods}

Three eyes, provided by the Minnesota Lions Eye Bank, were used in these tests; the eyes were designated as Nos 001, 002, and 003. The choroid was accessed by removing a $2.5 \mathrm{~mm}$ diameter disc from each sclera using a standard ophthalmic trephine. The experimental apparatus is shown in Figure 1. The IOP within the anterior chamber was controlled by changing the height of a manometer (a $60 \mathrm{ml}$ syringe connected to a 27 gauge needle) filled with $0.9 \%$ sodium chloride irrigation fluid. A second 27 gauge needle, connected to a pressure transducer (a disposable DTX/Plus

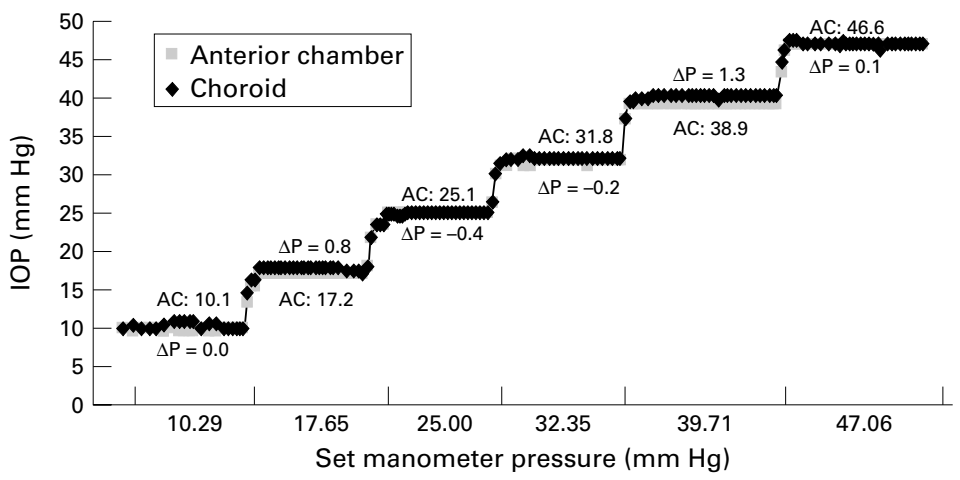

Figure 2 Data acquisition system output of the measured anterior chamber and choroid (fixed sensor) intraocular pressures (IOP) versus the set manometer pressure for eye No 001 . system from Becton Dickinson) was used to monitor the anterior chamber IOP. Both needles were inserted into the anterior chamber through the cornea. A CP-302 preamplifier, from Sable Systems Inc, was used to power the pressure transducer and to filter (low pass, $<1 \mathrm{~Hz})$ and amplify $(\times 100)$ the output. The amplified signal was then sampled at 0.5 $\mathrm{Hz}$ using an XR440-M data logger (data acquisition system) from Pace Scientific. The pressure at the choroid was monitored using an EPI-411-5P/R sensor, from Entran Devices, Inc, and the output was filtered, amplified, and stored as described above. The sensor was placed in intimate contact with the choroid, perpendicular to the eye surface, and held in place using a three dimensional miniature positioning stage from Daedal/Parker. The sensor was placed against the choroid at depths ranging from $0-2 \mathrm{~mm}$ below the scleral surface. It was observed that as long as there was some initial contact, the pressures measured by the Entran sensor were relatively insensitive to the sensor depth.

Initially, the sensors were powered and allowed to stabilise for about 30 minutes, minimising thermal drift. The two 27 gauge needles were then inserted and the manometer was set to $14 \mathrm{~cm} \mathrm{H}_{2} \mathrm{O}(10.3 \mathrm{~mm} \mathrm{Hg})$ above the eye pressure. The Entran transducer was then positioned against the choroid and allowed to stabilise over a 3-10 minute period in order to reach thermal equilibrium. The pressure in the eye was adjusted from $10.3 \mathrm{~mm} \mathrm{Hg}$ to 47.1 $\mathrm{mm} \mathrm{Hg}$ in steps of $7.35 \mathrm{~mm} \mathrm{Hg}$; each pressure level was held for approximately 45-60 seconds. All of the measurements were taken within a 12 hour period. In order to compare the pressure readings in each of the three eyes, the Entran sensor output was set to zero, for each experiment, at the initial anterior chamber pressure of $10.3 \mathrm{~mm} \mathrm{Hg}$. 


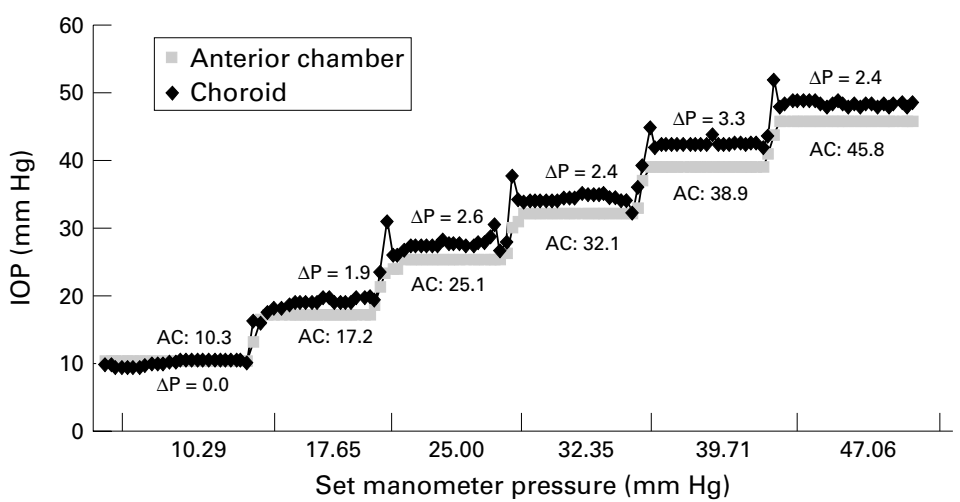

Figure 3 Data acquisition system output of the measured anterior chamber and choroid (sutured sensor) intraocular pressures (IOP) versus the set manometer pressure for eye 001.

Table 1 Differences between choroid and anterior chamber $(A C)$ pressures $(\triangle P)$ for each eye; and standard deviations (SD) over time of the choroid and anterior chamber pressures

\begin{tabular}{|c|c|c|c|c|c|c|c|}
\hline & $\begin{array}{l}\Delta P_{\min } \\
(m m \mathrm{Hg})\end{array}$ & $\begin{array}{l}\Delta P_{\max } \\
(m m \mathrm{Hg})\end{array}$ & $\begin{array}{l}\Delta P_{\text {avg }} \\
(m m \mathrm{Hg})\end{array}$ & $\begin{array}{l}\text { Measurement } \\
\text { location }\end{array}$ & $\begin{array}{l}S D_{\min } \\
(m m \mathrm{Hg})\end{array}$ & $\begin{array}{l}S D_{\max } \\
(m m \mathrm{Hg})\end{array}$ & $\begin{array}{l}S D_{\text {avg }} \\
(m m \mathrm{Hg})\end{array}$ \\
\hline \multirow[t]{2}{*}{ Eye 001} & \multirow[t]{2}{*}{-0.4} & \multirow[t]{2}{*}{1.3} & \multirow[t]{2}{*}{0.5} & Choroid & 0.24 & 0.48 & 0.31 \\
\hline & & & & AC & 0.00 & 0.50 & 0.23 \\
\hline \multirow[t]{2}{*}{ Eye 002} & \multirow[t]{2}{*}{-2.7} & \multirow[t]{2}{*}{0.2} & \multirow[t]{2}{*}{1.4} & Choroid & 0.00 & 0.71 & 0.30 \\
\hline & & & & $\mathrm{AC}$ & 0.00 & 0.38 & 0.11 \\
\hline \multirow[t]{2}{*}{ Eye 003} & \multirow[t]{2}{*}{-0.1} & \multirow[t]{2}{*}{1.5} & \multirow[t]{2}{*}{0.4} & Choroid & 0.23 & 0.73 & 0.48 \\
\hline & & & & $\mathrm{AC}$ & 0.00 & 0.44 & 0.23 \\
\hline \multirow{2}{*}{$\begin{array}{l}\text { Eye } 001 \\
\quad \text { (sutured) }\end{array}$} & \multirow[t]{2}{*}{0.0} & \multirow[t]{2}{*}{3.3} & \multirow[t]{2}{*}{2.1} & Choroid & 0.34 & 0.99 & 0.64 \\
\hline & & & & AC & 0.00 & 0.37 & 0.06 \\
\hline
\end{tabular}

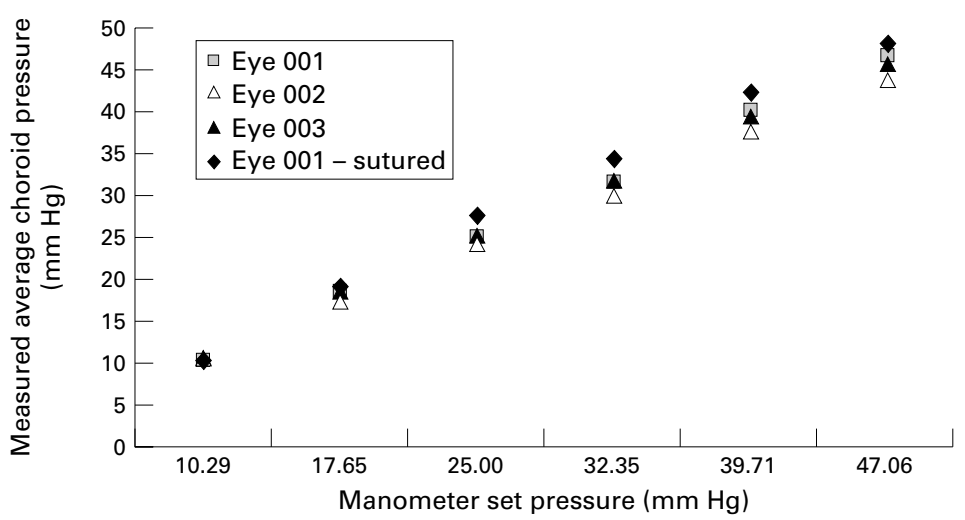

Figure 4 A comparison of the measured average choroid pressure versus the set manometer pressure for the three cadaver eyes.

Finally, in order to more closely replicate the final microsensor configuration, a second Entran pressure transducer was sutured to the sclera, as shown in Figure 1. The sensor was glued with epoxy to a thin plastic plate that was then sutured to the sclera. This "sutured sensor test" was conducted on eye No 001 and the experimental procedure described above for the fixed sensor was repeated.

\section{Results}

FIXED SENSOR

Figure 2 is a plot of the data acquisition system output for the IOP measured at the anterior chamber and at the choroid (using the fixed sensor configuration described earlier), versus the actual (manometer) pressure for eye No 001. The average anterior chamber pressure (averaged over 45-60 seconds), is denoted in the figure by AC, and varies from $10.1 \mathrm{~mm} \mathrm{Hg}$ (for a manometer pressure of $10.29 \mathrm{~mm} \mathrm{Hg}$ ) to $46.6 \mathrm{~mm} \mathrm{Hg}$ (for a manometer pressure of $47.06 \mathrm{~mm} \mathrm{Hg}$ ). The difference between the average anterior chamber pressure and the average choroid pressure is denoted in Figure 2 by $\Delta \mathrm{P}$. These readings vary from $\Delta \mathrm{P}_{\min }=-0.4$ $\mathrm{mm} \mathrm{Hg}$ to a maximum of $\Delta \mathrm{P}_{\text {max }}=1.3 \mathrm{~mm} \mathrm{Hg}$, with an average difference of $\Delta \mathrm{P}_{\text {avg }}=0.5 \mathrm{~mm}$ $\mathrm{Hg}$.

Results similar to those shown in Figure 2 were also obtained for eye Nos 002 and 003.

SUTURED SENSOR

Figure 3 is a plot of the data acquisition system output for the IOP measured at the anterior chamber and at the choroid (using the sutured sensor configuration described earlier), versus the manometer pressure for eye No 001. The difference between the average anterior chamber and choroid pressures varies from $\Delta \mathrm{P}_{\min }=$ $1.9 \mathrm{~mm} \mathrm{Hg}$ to a maximum of $\Delta \mathrm{P}_{\max }=3.3 \mathrm{~mm}$ $\mathrm{Hg}$ with an average of $\Delta \mathrm{P}_{\text {avg }}=2.1 \mathrm{~mm} \mathrm{Hg}$.

Table 1 summarises the results for all of the tests. The data include minimum, maximum, and average standard deviations for the measured choroid and anterior chamber pressures. The data show that the standard deviations for the anterior chamber pressure measurements for all test cases ranged from $\mathrm{SD}_{\min }=0.0 \mathrm{~mm} \mathrm{Hg}$ to $\mathrm{SD}_{\max }=0.50 \mathrm{~mm} \mathrm{Hg}$ with an average of $\mathrm{SD}_{\text {avg }}=0.16 \mathrm{~mm} \mathrm{Hg}$. The standard deviation for the fixed sensor choroid pressure measurements ranged from $\mathrm{SD}_{\min }=$ $0.00 \mathrm{~mm} \mathrm{Hg}$ to $\mathrm{SD}_{\max }=0.73 \mathrm{~mm} \mathrm{Hg}$ with an average of $\mathrm{SD}_{\text {avg }}=0.36 \mathrm{~mm} \mathrm{Hg}$. The standard deviation for the sutured sensor choroid pressure measurements ranged from $\mathrm{SD}_{\min }=$ $0.34 \mathrm{~mm} \mathrm{Hg}$ to $\mathrm{SD}_{\max }=0.99 \mathrm{~mm} \mathrm{Hg}$ with an average of $\mathrm{SD}_{\text {avg }}=0.64 \mathrm{~mm} \mathrm{Hg}$.

Finally, Figure 4 is a plot of the average choroid pressure measurements, at each of the manometer pressure settings, for all three fixed sensor tests conducted here. The sutured sensor test results are also included for comparison. The results suggest that the measurements are relatively repeatable.

\section{Discussion}

A study of the data shown in Figure 2 indicates that the anterior chamber pressure (measured by the Becton Dickinson transducer) very accurately (within plus or minus $1.2 \mathrm{~mm} \mathrm{Hg}$ ) tracks the actual pressure for the six fixed manometer pressures studied here. The pressure measured by the Entran sensor at the choroid interface also tracks the actual pressure well, although not quite as uniformly as the anterior chamber measurements. Since both transducers have similar performance characteristics, the difference in accuracy is attributed to the fact that the Entran transducer must measure the IOP indirectly, through the choroid membrane; whereas the Becton Dickinson transducer is in direct fluid contact with the aqueous humour in the anterior chamber.

Figure 3 suggests that the sutured sensor choroid pressure tracks the actual pressure almost as well as the fixed sensor does. The primary differences are the slightly noisier response, a larger initial offset, and a brief overshoot when changing pressure levels. The 
initial offset can be attributed to a lack of complete, intimate contact between the sensor surface and the choroid due to the large sensor size and suturing plate. The average pressure difference between the choroid IOP and the anterior chamber IOP of $\Delta \mathrm{P}_{\text {avg }}=2.1 \mathrm{~mm} \mathrm{Hg}$ would drop significantly once the initial offset is accounted for.

The relatively small standard deviations and pressure differences shown in Table 1 suggest that, even with the relatively large choroid sensor used here (manufacturer specifications for non-linearity, hysteresis, and thermal drift, were of the order of 3-5 $\mathrm{mm} \mathrm{Hg}$ ), changes in IOP on the order of $1 \mathrm{~mm} \mathrm{Hg}$ could easily be detected at the choroid interface with a more sensitive sensor.

\section{Conclusions}

The tests described in this paper have shown that small changes in IOP can be readily detected by a sensor that is inserted through a hole trephined into the sclera and is placed in direct contact with the surface of the choroid.
Accurate pressure tracking was demonstrated for both a fixed sensor configuration and for a sensor sutured to the sclera. These results are the first step in the realisation of a surgically implantable microsensor transponder to remotely monitor IOP for patients suffering from difficult to manage glaucoma.

The authors would like to thank Drs Richard Brubaker and Jay W McLaren of the Ophthalmology Department of the Mayo Clinic and Foundation for their encouragement and technical support throughout this work. We also wish to thank Professors Dennis Polla and Arthur Erdman, of the University of Minnesota. This work was supported in part by funding from an internal grant from the Biomedical Engineering Department at the University of Minnesota.

1 Davson H. Physiology of the eye. New York: Pergamon Press, 1990.

2 Collins CC. Miniature passive pressure transensor for implanting in the eye. IEEE Trans Biomed Eng 1967;14:7483 .

3 Green, ME. Intraocular pressure measurement with instrumented contact lenses. Invest Ophthalmol Vis Sci 1974;13: 299-302.

4 Cooper RL, Beale DG. Radio telemetry of intraocular pressure in vitro. Invest Ophthalmol Vis Sci 1977;16:168-71.

5 McLaren JW, Brubaker RF, FitzSimon JS. Continuous measurement of intraocular pressure in rabbits by telemetry. Invest Ophthalmol Vis Sci 1996;37:966-75. 